\title{
Pensar, gostar e considerar importante a prática de atividade física aumentam as chances de ser ativo no lazer?
}

\author{
Do thinking, enjoying, and considering important the practice of physical activity \\ increase the chances of being active at leisure?
} Elaynne Silva de Oliveira ${ }^{1}$ (D) Claudia Vanisse de Brito Costa ${ }^{1,2}$ (D), Sergio Augusto Rosa de Souza ${ }^{3}$ (D),
Wellington Roberto Gomes de Carvalho ${ }^{1,4}$ (D), Emanuel Péricles Salvador 2,3 (D)

\begin{abstract}
RESUMO
Embora reconhecida como comportamento de extrema importância na saúde da população, a prática de atividade física regular ainda é um grande desafio. Objetivo: verificar o nível de atividade física no lazer e possíveis fatores correlatados em uma comunidade acadêmica do nordeste brasileiro. Metodologia: trata-se de um estudo transversal, constituído por discentes, docentes e técnicos administrativos, utilizou-se como instrumento "Mapa de Atividade Física e Saúde-MAFIS", realizou-se estatística descritiva, teste de Qui quadrado, com posterior montagem do modelo de regressão logística. Resultados: $52,1 \%$ atingiram as recomendações de atividade física (AF), os homens apresentam 65,3\% (OR: 1,65; IC95\%: 1,29-2,11) mais chance de serem ativos, quem gosta de AF tem duas (OR: 2,02; IC95\%: 0,82-4,96) a 4,8 (OR: 4,84; IC95\%: 1,99-11,8) vezes mais chance de praticar AF, achar importante fazer AF (OR: 2,23; IC95\%: 0,48-10,4) e menos dias sentado aumenta de 48,3\% (OR: 1,48; IC95\%: 1,032- 2,130) a 53,3\% (OR: 1,53; IC95\%: 1,09-2,13) a chance de fazer AF e quem pensa em fazer AF (OR: 0,362; IC: 0,203-0,645) tem menos chance de ser ativo. Conclusão: Gostar, considerar importante e menos dias sentados aumentam as chances de fazer atividade física, já pensar sempre em fazer, diminui a chance de ser ativo no lazer.
\end{abstract}

Palavras-chave: Exercício Físico; Saúde; Universidades; Estudantes; Docentes.

\begin{abstract}
Although recognized as extremely important behavior in the health of the population, the practice of a regular physical activity is still a major challenge. Aim: verifying the level of leisure-time physical activity and possible correlated factors in an academic community in northeastern Brazil. Materials and methods: this is a cross-sectional study, consisting of students, professors, and administrative technicians. The instrument "Map of Physical Activity and Health-MAFIS" was used, as well as descriptive statistics, Chi-square test, with the subsequent assembly of the logistic regression model. Results: $52.1 \%$ reached the recommendations for physical activity (PA), men were $65.3 \%$ (OR: $1.65 ; 95 \% \mathrm{CI}: 1.29-2.11$ ) more likely to be active, those who like PA were two (OR: $2.02 ; 95 \%$ CI: $0.82-4.96$ ) to 4.8 (OR: $4.84 ; 95 \%$ CI: $1.99-11.8$ ) times more likely to practice PA, finding it important to do AF (OR: $2.23 ; 95 \% \mathrm{CI}: 0.48-10.4$ ) and spending fewer days sitting increased by $48.3 \%$ (OR: $1.48 ; 95 \% \mathrm{CI}: 1,032-2,130$ ) to $53.3 \%$ (OR: 1,$53 ; 95 \%$ CI: $1.09-2.13$ ) the chance of doing PA and those who think about doing PA (OR: 0.362 ; CI: $0.203-0.645$ ) are less likely to be active. Conclusion: Enjoying, considering important, and spending fewer days sitting increase the chances of doing physical activity, on the other hand, just thinking about doing it decreases the chances of being active at leisure.
\end{abstract}

Keywords: Physical Activity; Health; Universities; Students; Professor, University.

Programa de Pós- Graduação Nutrição em Saúde Pública, Universidade de São Paulo (PPGNSP-USP), São Paulo, Brasil.

Programa de Pós-Graduação em Educação Física da UFMA, São Luis (MA), Brasil.

Departamento de Educação Física, UFMA, São Luis (MA), Brasil.

Universidade Federal de Uberlândia, Uberlândia (MG), Brasil.

$\triangle$ Elaynne Silva de Oliveira. Universidade Federal do Maranhão, Departamento de Educação Física, Núcleo de Esportes. Av. dos Portugueses, 1966 - Vila Bacanga. CEP: 65080-805. São Luís (MA), Brasil.

elaynneedf@gmail.com | Recebido em: 15/06/2020 | Aprovado em: 10/08/2020 


\section{INTRODUÇÃO}

A atividade física (AF) já é reconhecida como um dos principais fatores para adoção de um estilo de vida saudável ${ }^{1}$. No aspecto individual, a atividade física está associada a maior capacidade de trabalho, mais entusiasmo para viver e sensação de bem-estar. Em grandes populações está associada a menores gastos de saúde, diminuição da mortalidade, assim como menor risco de desenvolver doenças crônicas como hipertensão, diabetes mellitus tipo 2 e obesidade 2 . A Organização Mundial de Saúde recomenda que adultos entre 18 a 64 anos pratiquem pelo menos de 75 a 150 minutos de AF semanais de intensidade vigorosa a moderada ${ }^{3}$. Apesar de todos os benefícios e diferentes possibilidades, ainda é elevado o número de pessoas que não atingem o mínimo recomendado de $\mathrm{AF}^{4}$.

Embora reconhecida como comportamento de extrema importância na saúde da população, a prática de atividade física regular ainda é um grande desafio. A prevalência de inatividade física no mundo é de $31,1 \%$, com variações entre os continentes. Diferenças também foram encontradas quanto ao gênero, com mulheres inativas alcançando 33,9\% da população total e os homens alcançaram $27,9 \%{ }^{4}$. No Brasil, seis em cada dez pessoas $(62,1 \%)$ com 15 anos ou mais não praticam esporte e/ou atividade física, são mais de 100 milhões de sedentários ${ }^{5}$.

No contexto das instituições de ensino superior, diversos estudos têm relatado altas prevalências de inatividade física em comunidade acadêmica6-8. A vida universitária, para os que compõem a comunidade acadêmica, em especial para o discente, é marcada por grandes mudanças na vida psicossocial, profissional e nos comportamentos de saúde ${ }^{9}$. Tais fatores podem contribuir para mudanças no estilo de vida, seja de forma positiva ou negativa ${ }^{10,11}$. Mesmo se tratando de uma população que sofre diversas influências, nota-se uma lacuna e ausência de estudos que contemplem todos que formam a comunidade universitária ${ }^{12-15}$.

Tendo em vista que os hábitos adquiridos na universidade, embora contemplem um período temporário, podem ser presentes muito além dessa época. Além das altas prevalências de fatores de risco à saúde, é de grande importância o conhecimento adquirido sobre os benefícios de um estilo de vida fisicamente ativo e identificar comportamentos correlatados a saúde na comunidade universitária representa o início de tomadas de decisões para se propor políticas e estratégias nesta população. Assim, o presente estudo tem como objetivo verificar o nível de atividade física no lazer e possíveis fatores correlatados em comunidade acadêmica do nordeste brasileiro.

\section{MÉTODOS}

\section{Delineamento e participantes}

Trata-se de um estudo transversal, com uma amostra não probabilística, constituída por discentes, docentes e técnicos administrativos referentes ao banco de dados do primeiro ano de coleta da pesquisa longitudinal "Mover pela Saúde", que visa avaliar no período de dez anos o nível de atividade física e estilo de vida da comunidade acadêmica da Universidade Federal do Maranhão, aprovado pelo Comitê de Ética e Pesquisa com Seres Humanos, sob o parecer $n^{\circ} 2.335 .729$. Todos os participantes foram informados sobre os objetivos e procedimentos aos quais seriam submetidos e assinaram o termo de consentimento livre e esclarecido (TCLE).

Para cálculo da amostra adotou-se a técnica de amostragem com correção para populações finitas e recorreu-se a equação proposta por Luiz e Magnanini ${ }^{16}$, com o nível de confiança de $95 \%$ e erro tolerável de 3,5 pontos percentuais. Considerando a falta de pesquisas anteriores na população estudada e a frequência dos desfechos a serem investigados, assim como a característica do levantamento de diferentes informações, foi adotada uma proporção de $50 \%$. Chegou-se a um cálculo de 728 sujeitos, com acréscimo de $20 \%$ para possíveis perdas, finalizando em 874 sujeitos.

\section{Procedimentos}

A coleta de dados ocorreu de abril a dezembro de 2018, sob realização e organização dos membros do Laboratório de Estudos e Pesquisas Epidemiológicas em Atividade Física e Esporte e Saúde-LAPAES. Todos os que compõem a comunidade acadêmica foram convidados a participar do estudo. Foram excluídos apenas as pessoas que 
por algum motivo não tinham acesso à internet. A divulgação foi realizada em site e redes sociais oficiais da universidade, e por meio de visitas itinerantes aos cursos de graduação, centros acadêmicos, departamentos, setores administrativos, entre outros, mediante a autorização dos professores e chefes de departamento.

Utilizou-se como instrumento o questionário online intitulado "Mapa de Atividade Física e SaúdeMAFIS" disponível no link https://mafis.herokuapp. $\mathrm{com} /$, com blocos em processo de validação, compostos por 97 perguntas divididos em 12 blocos (1- Identificação; 2-Nível de Atividade física; 3-Tendência para mudança no nível de atividade física; 4-Preferência para atividade física; 5-Risco de doença cardiovascular; 6-Risco associado à obesidade; 7-Dor e doenças crônicas não transmissíveis; 8-Nível de estresse; 9- Tendência de mudança no nível de estresse; 10-Padrão alimentar; 11-Tendência de mudança do padrão alimentar; 12-Auto-eficácia.

Cada bloco avaliado gera um relatório que classifica o indivíduo de acordo com os valores de referência conhecidos na literatura, esses relatórios foram visualizados ao término de cada bloco ou enviados ao e-mail automaticamente ao final da participação do indivíduo. Por se tratar de um instrumento dividido por blocos, os participantes podiam responder os blocos de maior interesse, logo a amostra foi avaliada e apresentada pelo percentual válido, descrito nos resultados.

\section{Análise dos dados}

Utilizou-se como desfecho principal o nível de atividade física no lazer, elaborado a partir do escore de atividade física semanal, baseado nas recomendações de 150 minutos por semana propostos pela Organização Mundial de Saúde-OMS ${ }^{3}$, no qual foi calculada frequência semanal (dias) pela duração média (minutos) multiplicada pelo peso da atividade ( 1 = leve a moderada; 2 = moderada a vigorosa; $1,5=$ que se encaixem nos dois tipos), sendo os sujeitos classificados, após os cálculos, de acordo com o escore obtido em minutos, no qual, a) = 0 a $<150$ minutos/semana - não atende as recomendações; b) $\geq 150$ minutos/semana- atendem as recomendações.
Como desfecho secundário utilizou- se as variáveis dicotômicas e categóricas: idade $(p=0,05)$, situação conjugal $(p=0,024)$, consumo semanal de frutas $(p=0,13)$, consumo de hortaliças $(p=0,05)$, consumo de biscoitos recheados $(p=0,023)$, disposição ao acordar $(p=0,031)$, sensação de estresse $(p=0,004)$, gostar de fazer AF $(p=0,000)$, considera importante fazer $\operatorname{AF}(p=0,000)$, pensa em fazer AF $(p=0,019)$, dias sentados por mais de três horas $(p=0,000)$, percepção de humor $(p=0,003)$ e sexo $(p=0,000)$.

A análise dos dados foi realizada através de estatística descritiva em forma de frequência absoluta e percentual. O teste Qui-quadrado foi utilizado para verificar as possíveis associações entre as variáveis dependente e independente relacionadas ao estilo de vida. Para a montagem da regressão logística utilizou-se apenas as variáveis que apresentaram valor de $p<0,20$. Estas foram ordenadas de maneira crescente no modelo de acordo com o valor de significância e foram construídas estimativas de razão de chances (OR) e intervalos com 95\% de confiança (IC 95\%). O nível de significância adotado foi de $5 \%$, e o programa SPSS, versão 23.0 foi utilizado.

\section{RESULTADOS}

A amostra do estudo foi de 2465 participantes, a Tabela 1 apresenta as características sociodemográficas e comportamentais dos participantes. A maior parte da amostra é do sexo feminino $58,9 \%$, cor de pele parda $54 \%$, solteiros $61 \%$, em estado nutricional classificado como peso normal $56,2 \%$ e pertencente a faixa etária até 29 anos 70,6\%. Com relação à prática de $\mathrm{AF}$ no tempo livre, 52,1\% atingiram as recomendações mínimas de atividade física para benefícios à saúde e $47,9 \%$ foram considerados insuficientemente ativos. Com relação à renda, 62,3\% recebem de 1 a 3 salários mínimos.

De acordo com o modelo de regressão logística binária na Tabela 2, os homens apresentam 65,3\% (OR: 1,65; IC95\%: 1,29-2,11) mais chance de serem ativos quando comparados com as mulheres. Quem respondeu que "gosta um pouco" de AF tem duas vezes (OR: 2,02; IC95\%: $0,82-4,96)$ mais chance de fazer AF no tempo livre em comparação com quem "não gosta". E quem 
"gosta muito", possui 4,8 vezes (OR: 4,84; IC95\%: $1,99-11,8)$ mais chance de praticar AF em comparação com quem não gosta.

Em relação a achar importante fazer $A F$, à medida que a pessoa considera praticar AF mais importante, aumenta a chance de fazer AF (OR: 2,23; IC95\%: 0,48-10,4).

À proporção que passam menos dias sentados por mais de três horas vendo TV, de frente para o computador, aumenta a chance de fazer AF no tempo livre. Quem fica de três a quatro dias sentado aumenta em 53,3\% (OR: 1,53; IC95\%: 1,09-2,13) em comparação a quem fica de cinco a sete dias. E quem fica de zero a dois dias aumenta 48,3\% (OR: 1,48; IC95\%: 1,032-2,130). No domínio do "pensar em fazer AF", quem "pensa sempre em fazer AF" (OR: tem menos chance de fazer em comparação com quem não pensa.

\section{Tabela 1}

Características da amostra da comunidade acadêmica, São Luís, MA, Brasil, 2018.

\begin{tabular}{|c|c|c|c|}
\hline Variáveis & & $n$ & $(\%)$ \\
\hline \multirow{2}{*}{ Nível de AF no lazer } & Não alcança as recomendações & 642 & 47,9 \\
\hline & Alcança as recomendações & 697 & 52,1 \\
\hline \multirow[t]{2}{*}{ Sexo } & Feminino & 1453 & 58,9 \\
\hline & Masculino & 1012 & 41,1 \\
\hline \multirow[t]{5}{*}{ Idade } & Até 29 anos & 1667 & 70,6 \\
\hline & 30-39 anos & 372 & 15,8 \\
\hline & 40- 49 anos & 221 & 9,4 \\
\hline & 50-59 anos & 72 & 3,1 \\
\hline & 60 ou mais & 28 & 1,2 \\
\hline \multirow[t]{2}{*}{ Escolaridade } & $\begin{array}{l}0-12 \text { anos de estudo } \\
13-17 \text { anos de estudo }\end{array}$ & $\begin{array}{c}159 \\
2238\end{array}$ & $\begin{array}{c}6,5 \\
90,8\end{array}$ \\
\hline & $>18$ anos de estudo & 68 & 2,8 \\
\hline \multirow[t]{4}{*}{ Situação conjugal } & Casado & 462 & 18,7 \\
\hline & Divorciado & 52 & 2,1 \\
\hline & Namorando & 448 & 18,2 \\
\hline & Solteiro & 1503 & 61 \\
\hline \multirow[t]{5}{*}{ Renda } & 1 a 3 salários mínimos & 1142 & 62,3 \\
\hline & 4 a 9 salários mínimos & 412 & 22,5 \\
\hline & 10 a 15 salários mínimos & 106 & 5,8 \\
\hline & > 15 salários mínimos & 49 & 2,7 \\
\hline & Não quer informar & 123 & 6,7 \\
\hline \multirow[t]{4}{*}{ IMC } & Abaixo do peso & 99 & 8,1 \\
\hline & Peso normal & 689 & 56,2 \\
\hline & Sobrepeso & 318 & 26 \\
\hline & Obesos & 119 & 9,7 \\
\hline \multirow[t]{2}{*}{ Fuma } & Não & 1308 & 96 \\
\hline & Sim & 54 & 4 \\
\hline \multirow[t]{2}{*}{ Álcool } & Não & 683 & 58,6 \\
\hline & Sim & 482 & 41,4 \\
\hline \multirow[t]{5}{*}{ Dias sentado $3 \mathrm{~h}$ tempo livre } & 1-2 dias sentados & 805 & 38 \\
\hline & 3-4 dias sentados & 381 & 18 \\
\hline & 5-6 dias sentados & 279 & 13,2 \\
\hline & 7 dias sentados & 425 & 20 \\
\hline & Nenhum & 231 & 10,8 \\
\hline \multirow[t]{3}{*}{ Considera importante fazer AF no tempo livre } & É muito importante, mas não prioridade & 988 & 54 \\
\hline & É muito importante, prioridade & 816 & 44,5 \\
\hline & Não é importante & 27 & 1,5 \\
\hline \multirow[t]{3}{*}{ Gosta de fazer AF no tempo livre } & Gosto muito & 1055 & 57,7 \\
\hline & Gosto pouco & 644 & 35,2 \\
\hline & Não gosto & 130 & 7,1 \\
\hline \multirow[t]{3}{*}{ Pensa em fazer ou fazer mais AF no tempo livre } & Penso sempre & 1265 & 69,1 \\
\hline & Penso às vezes & 481 & 26,3 \\
\hline & Não penso & 86 & 4,7 \\
\hline Total & & & $100 \%$ \\
\hline
\end{tabular}

IMC: índice de massa corporal; AF: atividade física 
Tabela 2

Regressão logística binária entre os fatores associados e AF no lazer, São Luís, MA, Brasil, 2018.

\begin{tabular}{|c|c|c|c|}
\hline Variáveis & p valor & OR & IC $95 \%$ \\
\hline Sexo & $0,000 *$ & 1,65 & $(1,29-2,11)$ \\
\hline Não gosta de AF & $0,000 *$ & & \\
\hline Gosta pouco de AF & 0,124 & 2,02 & $(0,82-4,96)$ \\
\hline Gosta muito de AF & $0,001 *$ & 4,84 & $(1,99-11,80)$ \\
\hline Não considera importante AF & $0,003 *$ & & \\
\hline Considera importante, não prioridade & 0,639 & 1,44 & $(0,31-6,66)$ \\
\hline Considera muito importante, uma prioridade & 0,305 & 2,23 & $(0,48-10,43)$ \\
\hline Dias sentado no tempo livre (5-7) & $0,035^{*}$ & & \\
\hline Dias sentado no tempo livre $(3-4)$ & $0,012 *$ & 1,53 & $(1,09-2,13)$ \\
\hline Dias sentado no tempo livre $(0-2)$ & $0,033 *$ & 1,48 & $(1,03-2,13)$ \\
\hline Não pensa em fazer AF & $0,000 *$ & & \\
\hline Pensa às vezes em fazer AF & 0,074 & 0,57 & $(0,31-1,05)$ \\
\hline Pensa sempre em fazer AF & $0,001 *$ & 0,36 & $(0,20-0,64)$ \\
\hline Constante & 0,087 & 0,22 & \\
\hline
\end{tabular}

* $p$-valor < 0,005 para teste de associação com atividade física

AF: atividade física.

Ajustada por sexo.

\section{DISCUSSÃO}

O objetivo principal deste estudo foi verificar o nível de atividade física no lazer e possíveis fatores correlatados em comunidade acadêmica do nordeste brasileiro. Verificou-se como principais achados que mais de $52,1 \%$ da amostra atingiu as recomendações de atividade física, os homens apresentaram mais chance de serem ativos, assim como as pessoas que gostam, consideram importante fazer atividade física e passam menos dias sentados. Já comportamentos como pensar em fazer AF diminui as chances de ser ativo no lazer.

Os resultados apontam que mais da metade dos indivíduos praticavam 150 minutos ou mais de atividade física de lazer por semana. Prevalências aceitáveis quando comparadas a outros estudos que evidenciam elevados níveis de inativos fisicamente, é o caso da Pesquisa Nacional de Amostra por Domicílios-PNAD, que demonstra que no Brasil seis em cada dez pessoas $(62,1 \%)$ com 15 anos ou mais não praticaram esporte e/ou atividade física, representando mais de 100 milhões de sedentários ${ }^{5}$. Indo de encontro aos nossos achados, um estudo realizado com a comunidade acadêmica (discentes e docentes) da Universidade de São Paulo ${ }^{17}$ relatou elevadas prevalências de ativos entre homens $(70,7 \%)$ e mulheres $(72,9 \%)$. Pode-se supor que esses achados estejam relacionados ao fato de existir uma associação entre escolaridade e atividade física. Um estudo de revisão sistemática ${ }^{18}$ com o objetivo de verificar a associação entre os níveis de prática de atividades físicas e as condições socioeconômicas em adultos e idosos constatou que em $16(66,6 \%)$ dos 43 estudos selecionados houve uma relação positiva. Pessoas com maior escolaridade estavam mais propensos a fazerem atividade física no tempo de lazer, o que pode contribuir para justificar nossos achados, haja vista a amostra ser em sua maioria de pessoas em formação ou formadas com nível superior.

Já em relação ao sexo, os resultados revelaram uma prevalência maior de atividade física no lazer (AFL) entre os homens quando comparados com as mulheres, eles possuem $65,3 \%$ mais chances de serem ativos. Esses dados confirmam a hipótese de que homens são mais fisicamente ativos ${ }^{19}$ e vai ao encontro do verificado em estudos semelhantes ${ }^{20,21}$.

A atividade física é entendida como um fenômeno de dimensões biológicas e culturais, inerente ao ser humano, que representa um determinante de saúde complexo, multifatorial e de difícil mudança nas populações ${ }^{22}$. Segundo um estudo de Baumam et al. ${ }^{23}$, existem mais de 70 variáveis que se associam a prática de atividade física em populações que vivem em países de baixa e média renda, incluindo variáveis biológicas, sociais, comportamentais e ambientais. No presente estudo foi 
possível verificar que as variáveis gostar, pensar, considerar importante, assim como dias sentados aumentam ou diminuem as chances de fazer atividade física no lazer.

De acordo com a regressão logística, as pessoas que declararam "gostar um pouco" de AF tem duas vezes mais chance de fazer AF no tempo livre e quem "gosta muito", possui 4,8 vezes mais chance em comparação com quem não gosta. Corroborando com o estudo, Mielke et al. ${ }^{24}$ realizaram uma pesquisa na Universidade Federal de Pelotas com uma amostra de 485 universitários de 17 cursos de graduação que apresentou resultados semelhantes. Aqueles que relataram gosto pela AFL tiveram sua chance aumentada em quatro vezes, quando comparados aqueles que declararam não gostar. Segundo os resultados da pesquisa por amostra de domicílios do IBGE$^{5}$, "não gostar" está entre um dos principais motivos que levam as pessoas a não praticarem atividade física ou esporte. Tais evidências contribuem para explicar como a variável "gostar" se associa a fazer ou não atividade física.

Assim como gostar, considerar importante também aumenta em 2,2 vezes a chance de praticar atividade física, quando comparado a quem não considera importante e uma prioridade. Já se tem na literatura evidências demonstrando a importância da atividade física e comportamentos saudáveis na saúde de indivíduos e populações ${ }^{25}$. Segundo resultados do Diagnóstico Nacional do Esporte, $80,4 \%$ das pessoas que são sedentárias alegam conhecer os riscos de tais comportamentos e $69,8 \%$ citam que os principais motivos para o abandono da prática de atividade física ou esporte se relaciona a falta de tempo e outras prioridades como estudo, trabalho e família ${ }^{26}$. Assim, a partir dos nossos achados, supõe-se que reconhecer ser importante fazer AF aumenta a chance do indivíduo ser ativo, e o inverso, que não ter $\mathrm{AF}$ como prioridade influencia diretamente na escolha de não praticar $\mathrm{AF}$ ou da prática em níveis insatisfatórios.

Resultados diferentes foram encontrados na associação da variável pensar em fazer atividade física. Evidenciou-se uma associação inversa, quem pensa sempre em fazer AF tem menos chance de fazer em comparação com quem não pensa. $\mathrm{Na}$ literatura foi possível encontrar uma relevante explicação para o achado, representado pelos estudos de Prochaska ${ }^{27}$ que propõe o Modelo Transtéorico de Mudança de Comportamento. Neste modelo organizado em estágios (pré-contemplação, contemplação, ação e manutenção), o fato de pensar classifica o indivíduo no nível de contemplação, a pessoa admite ter um problema e considera possibilidades de mudança, mostrando preocupação e uma clara avaliação entre as vantagens e as desvantagens de mudar, podendo permanecer por longos períodos na contemplação, substituindo a ação pelo pensamento ${ }^{28}$. Assim os indivíduos até pensam em fazer atividade física, mas, no entanto, seguem sem fazer e não chegam a ter uma atitude, ou mesmo, não apresentam motivação alguma para iniciar uma rotina de atividade física ${ }^{29}$.

O tempo sentado também já é descrito em trabalhos como um fator que está associado ao nível de atividade física e apresentado como um bom preditor para avaliar comportamentos sedentários ${ }^{30}$. Em nossos achados, quem passa menos dias sentado por mais de três horas vendo TV, de frente para computador, aumenta a chance de fazer AF no tempo livre. Um estudo realizado por Couto et al. ${ }^{31}$ com universitários de Brasília também encontrou uma associação positiva entre tempo sentado e o nível de atividade física. Acredita-se que por se tratar de uma amostra composta na sua maioria por universitários, esse comportamento esteja interligado a elevada carga de trabalhos e compromissos acadêmicos, participação em projetos de extensão e outras atividades curriculares.

O presente estudo apresenta pontos fortes e limitações que precisam ser mencionados: como principal limitação, cita-se o delineamento tranversal que não permite avaliar a relação causa efeito entre a exposição e desfecho, no entanto, vale ressaltar que esta investigação apresenta os principais dados da linha de base do estudo longitudinal que irá avaliar atividade e física e estilo de vida da comunidade acadêmica no período de dez anos. Entre os pontos positivos, ressalta-se a utilização de um instrumento (questionário online) criado especificamente para ser utilizado em ambiente virtual, ferramenta esta que se apresenta de forma promissora para diminuir o custo financeiro em coletas de dados e aumentar o alcance de sujeitos em pesquisas epidemiológicas. Outro aspecto positivo se 
refere ao conjunto maior de variáveis utilizadas no instrumento, o que do ponto de vista da epidemiologia é recomendado para que o pesquisador tenha convicção da relação da variável com o desfecho.

\section{CONCLUSÃO}

Conclui-se que gostar e considerar importante fazer atividade física e menos dias sentados na semana, aumentam as chances das pessoas serem ativas. Já os sujeitos que pensam em fazer atividade física estão protegidos contra a prática no tempo de lazer. Acredita- se que os resultados deste estudo apresentam um conjunto de variáveis promissoras para compreensão de barreiras e facilitadores para a adoção de comportamentos ativos, dados estes relevantes que devem ser levados em consideração para nortear a construção de políticas no ambiente acadêmico. Por fim sugere-se a realização de mais estudos com todos que compõem a comunidade universitária.

\section{REFERÊNCIAS}

1. Ferreira JS, Diettrich SHC, Pedro DA. Influência da prática de atividade física sobre a qualidade de vida de usuários do SUS. Saúde debate. setembro de 2015;39(106):792-801.

2. Ding $D$, Lawson KD, Kolbe-Alexander TL, Finkelstein EA, Katzmarzyk PT, van Mechelen W, et al. The economic burden of physical inactivity: a global analysis of major non-communicable diseases. The Lancet. setembro de 2016;388(10051):1311-24

3. World Health Organization. Global recommendations on physical activity for health. [Internet]. 2010 [citado 11 de novembro de 2020]. Disponível em: http://www. ncbi.nlm.nih.gov/books/NBK305057/

4. Hallal PC, Andersen LB, Bull FC, Guthold R, Haskell W, Ekelund $U$. Global physical activity levels: surveillance progress, pitfalls, and prospects. The Lancet. julho de 2012;380(9838):247-57.

5. Instituto Brasileiro de Geografia e estatística. Pesquisa Nacional por Amostra de Domicílios: Práticas de esporte e atividade física-2015: Rio de Janeiro (RJ); 2017.

6. Concha-Cisternas $Y$, Guzmán-Muñoz E, Valdés-Badilla P, Lira-Cea C, Petermann F, Celis-Morales C. Factores de riesgo asociados a bajo nivel de actividad física y exceso de peso corporal en estudiantes universitarios. Rev méd Chile. agosto de $2018 ; 146(8): 840-9$.
7. Pinto A, Claumann G, Cordeiro P, Felden E, Pelegrini A. Barreiras percebidas para a prática de atividade física entre universitários de Educação Física. Rev Bras Ativ Fís Saúde [Internet]. 1 de janeiro de 2017 [citado 11 de novembro de 2020];22(1). Disponível em: https://periodicos.ufpel.edu.br/ojs2/index.php/ RBAFS/article/view/8194

8. Takenaka TY, Pagin M, Neves LM,Santos AC, Santos GAB. Incidência de inatividade física e fatores associados em estudantes universitários. Rev Bras Ciênc Movimento 2016; 24(4): 55-62.

9. Usher W, Curran C. Predicting Australia's university students' mental health status. Health Promotion International. $1^{\circ}$ de abril de 2019;34(2):312-22.

10. Snedden TR, Scerpella J, Kliethermes SA, Norman RS, Blyholder L, Sanfilippo J, et al. Sport and Physical Activity Level Impacts Health-Related Quality of Life Among Collegiate Students. Am J Health Promot. junho de 2019;33(5):675-82.

11. Tassini CC, Val GR do, Candido S da S, Bachur CK. Assessment of the Lifestyle of University Students in the Healthcare Area Using the Fantastic Questionnaire. International Journal of Cardiovascular Sciences [Internet]. 2017 [citado 11 de novembro de 2020]; Disponível em: https://www.scielo.br/scielo.php?scrip$\mathrm{t}=\mathrm{sci}$ _arttext\&pid=S2359-56472017000200117

12. Gamboa Delgado EM. Sobrepeso y obesidad en estudiantes universitarios colombianos y su asociación con la actividad física: Overweight and obesity in Colombian college students and its association with physical activity. Nutrición Hospitalaria. fevereiro de 2015;(2):629-36

13. Peltzer K, Pengpid S. The association of dietary behaviors and physical activity levels with general and central obesity among ASEAN university students. AIMS public health. junho de 2017, 4(3): 301.

14. Khabaz MN, Bakarman MA, Baig M, Ghabrah TM, Gari MA, Butt NS, et al. Dietary habits, lifestyle pattern and obesity among young Saudi university students. JPMA. The Journal of the Pakistan Medical Association. 2017 Oct; 67(10):1541-1546.

15. Costa MAP da, Vasconcelos AGG, Fonseca M de JM da. Prevalence of obesity, overweight and abdominal obesity and its association with physical activity in a federal University. Rev bras epidemiol. junho de 2014;17(2):421-36.

16. Luiz RR, Magnani M. A lógica da determinação do tamanho da amostra em investigações epidemiológicas. Cadernos saúde coletiva 2000; 8(2): 9-28.

17. Maciel E da S, Sonati JG, Modeneze DM, Vasconcelos JS, Vilarta R. Consumo alimentar, estado nutricional e nível de atividade física em comunidade universitária brasileira. Rev Nutr. dezembro de 2012;25(6):707-18.

18. Rodrigues PF, Melo M, Assis M, Oliveira A. Condições socioeconômicas e prática de atividades físicas em 
adultos e idosos: uma revisão sistemática. Rev Bras Ativ Fís Saúde. $1^{\circ}$ de maio de 2017;22(3):217-32.

19. Simão $C B$, Nahas MV, Oliveira ESAD. Atividade física habitual, hábitos alimentares e prevalência de sobrepeso e obesidade em universitários da Universidade do Planalto Catarinense-UNIPLAC, Lages-SC. Rev Bras Ativ Fís Saúde. Setembro de 2012, 12(1): 3-12.

20. Acebes-Sánchez J, Diez-Vega I, Esteban-Gonzalo S, Rodriguez-Romo G. Physical activity and emotional intelligence among undergraduate students: a correlational study. BMC Public Health 2019,19(1):1241. doi: 10.1186/s12889-019-7576-5

21. Olfert MD, Barr ML, Charlier CC, Greene GW, Zhou W, Colby SE. Sex Differences in Lifestyle Behaviors among U.S. College Freshmen. nternational journal of environmental research and public health 2019,16(3):482. doi:10.3390/ijerph16030482

22. Giles-Corti B, King AC. Creating active environments across the life course: "thinking outside the square". British Journal of Sports Medicine. 3 de dezembro de 2008;43(2):109-13.

23. Bauman AE, Reis RS, Sallis JF, Wells JC, Loos RJ, Martin BW. Correlates of physical activity: why are some people physically active and others not? The Lancet. julho de $2012 ; 380(9838): 258-71$.

24. Mielke GI, Ramis TR, Habeyche EC, Oliz MM, Tessmer MGS, Azevedo MR, Hallal PC. Atividade física e fatores associados em universitários do primeiro ano da Universidade Federal de Pelotas. Rev Bras Ativ Fís Saúde. Setembro de 2010; 15: 57-64.
25. Andersen LB, Mota J, Di Pietro L. Update on the global pandemic of physical inactivity. The Lancet. setembro de $2016 ; 388(10051)$ :1255-6.

26. Ministério do Esporte (BR). Diesporte: diagnóstico nacional do esporte. O perfil do sujeito praticante ou não de esportes e atividades físicas da população brasileira [Internet]. Brasília: Ministério do Esporte; 2016 [citado 2020 fev 12]. 70 p. (Caderno 2). Disponível em: Disponível em: http://www.esporte.gov.br/diesporte/ diesporte_revista_2016.pdf

27. Prochaska JO, Norcross JC. Systems of psychotherapy: A transtheoretical analysis. Oxford: University Press; 2018.

28. Prochaska JO, Norcross JC, Diclemente CC. Changing for good. New York: WilUam Morrow and Company; 1994.

29. Prochaska JO, Marcus BH, Dishman RK. Advances in exercise adherence. The Transtheoretical Model: Applications to Exercise. Champaign, IL: Human Kinetics Publishers. 1994:161-80.

30. Fontes ACD, Vianna RPT. Prevalência e fatores associados ao baixo nível de atividade física entre estudantes universitários de uma universidade pública da região Nordeste - Brasil. Rev bras epidemiol. março de $2009 ; 12(1): 20-9$.

31. Couto DAC, Martin DRS, Molina GE, Fontana KE, Junqueira Jr LF, Porto LGG. Nível insuficiente de atividade física se associa a menor qualidade de vida e ao estudo noturno em universitários do Distrito Federal. Revista Brasileira de Ciências do Esporte. julho de 2019;41(3):322-30. 